\title{
Neonatal Testosterone Exposure Protects Adult Male Rats from Stroke
}

\author{
Rebecca W. Persky ${ }^{\mathrm{a}}$ Fudong Liu ${ }^{\mathrm{a}}$ Yan Xu ${ }^{\mathrm{a}}$ Gillian Weston ${ }^{\mathrm{a}}$ Stephanie Levy ${ }^{\mathrm{a}}$ \\ Charles E. Roselli $^{\mathrm{b}}$ Louise D. McCullough ${ }^{\mathrm{a}}$ \\ ${ }^{a}$ Department of Neuroscience and Neurology, University of Connecticut Health Center, Farmington, Conn., and \\ ${ }^{b}$ Department of Physiology and Pharmacology, Oregon Health and Science University, Portland, Oreg., USA
}

\section{Key Words}

Testosterone $\cdot$ Aromatase $\cdot$ Ischemic injury $\cdot$ Sex

dimorphism • Estradiol

\begin{abstract}
Background: Men have a higher stroke incidence compared to women until advanced age. The contribution of hormones to these sex differences has been extensively debated. In experimental stroke, estradiol is neuroprotective, whereas androgens are detrimental. However, prior studies have only examined the effects of acute treatment paradigms; therefore, the timing and mechanism by which ischemic sexual dimorphism arises are unknown. Methods: The effects of exogenous neonatal androgen exposure on subsequent injury induced by middle cerebral artery occlusion in adulthood in male rats were examined. Rats were administered vehicle (oil), testosterone propionate (TP) or the nonaromatizable androgen dihydrotestosterone (DHT) for 5 days after birth. At 3 months of age, a focal stroke was induced. Results: Testosterone-treated rats (but not DHTtreated animals) had decreased infarct volumes ( $20 \mathrm{vs.} 33 \%$, $p<0.05$ ) as well as increased estradiol levels ( $39.4 \mathrm{vs.} 18.6 \mathrm{pg} /$ $\mathrm{ml}, \mathrm{p}<0.0001)$ compared to oil-treated animals. TP-injected males had increased testicular aromatase (P450arom) levels (3.6 vs. $0.2 \mathrm{ng} / \mathrm{ml}, \mathrm{p}<0.0001$ ) compared to oil-treated males.
\end{abstract}

The level of X-linked inhibitor of apoptosis, the primary endogenous inhibitor of caspase-induced apoptosis, was increased in TP-treated rats compared with the oil-treated males. Conclusions: Neonatal exposure to exogenous testosterone upregulates testicular aromatase expression in male rats and leads to adult neuroprotection secondary to changes in serum estradiol levels and cell death proteins. This study suggests that early exposure to gonadal hormones can have dramatic effects on the response to adult cerebrovascular injury.

Copyright $\odot 2012$ S. Karger AG, Basel

\section{Introduction}

Stroke is the fourth leading cause of death in the US and is the primary cause of long-term adult disability. Stroke affects 15 million people worldwide, and is placing an increasing economic burden on the aging population [1]. Sex differences are seen in the epidemiology of ischemic stroke; males have significantly higher incidence prior to the age of 80 , then incidence climbs dramatically in women to almost double that of age-matched men [2,

R.W.P. and F.L. contributed equally to this work.

\section{KARGER}

Fax +4161306 1234

E-Mail karger@karger.ch

www.karger.com
(C) 2012 S. Karger AG, Basel

0028-3835/13/0973-0271\$38.00/0

Accessible online at:

www.karger.com/nen
Louise D. McCullough

Department of Neuroscience

263 Farmington Avenue

Farmington, CT 06032 (USA)

E-Maillmccullough@uchc.edu 
3]. Differences in stroke incidence and stroke mortality are also seen in neonatal and pediatric populations, suggesting ischemic sensitivity is programmed during early development. Boys have higher incidence and poorer outcomes after stroke compared to age-matched females [4, 5], and elevations in serum testosterone levels have been implicated as a contributing factor to this 'ischemia-sensitive' phenotype. Interestingly, this pattern reverses in aged populations. These sex differences have been recapitulated in the laboratory [6,7]. As these sex differences are seen in both early development and in the aged, when hormone levels are relatively equivalent between the sexes, ischemic sensitivity likely reflects a complex interaction between sex chromosome compliment, the organizational effects of prenatal steroid exposure, and later activational effects of gonadal steroids on the cerebrovascular system.

Estradiol's protective effects in ischemic stroke have been well documented in the preclinical literature [8]; however, the few studies examining the role of androgens in stroke have been somewhat contradictory [9]. In young adult animals, castration significantly reduces ischemic injury. Supplementation with testosterone or its nonaromatizable metabolite dihydrotestosterone (DHT) restored infarct volumes to levels seen in intact males. However, others have shown that testosterone or DHT supplementation can decrease infarct size, and that the effects of androgens are critically dependent on the age of the animal examined $[10,11]$. This is consistent with recent data obtained in clinical populations; higher testosterone levels were associated with an increased risk of thromboembolic events in pediatric populations [4], but low testosterone levels predicted incident stroke in older men. This suggests that low testosterone levels are associated with increased vascular risk in adult populations [5, $12,13]$. Testosterone plays a crucial role in the sexual differentiation of gonads as well as in the development of sexual dimorphic regions of the brain [14-17]. Androgens are particularly important during the perinatal periods as the levels and activity of aromatase (P450arom), the enzyme that converts testosterone to estradiol, leading to brain masculinization, are highest during this period. Baseline aromatase activity and expression are higher in male neurons [15]. Ischemia leads to an increase in brain aromatase expression in both sexes possibly reflecting an endogenous protective mechanism [18].

Gonadal steroids also act in a different chromosomal environment in males and females (XX vs. XY). Several recent studies have demonstrated that the molecular cell death pathways activated in response to ischemic injury differ based on the sex of the animal, or cell examined [reviewed in 19]. Male neurons are exquisitely sensitive to cell death induced by molecules such as poly ADP-ribose polymerase (PARP-1), a key DNA repair enzyme activated by stroke which triggers the release of mitochondrial apoptosis-inducing factor (AIF). Females in contrast, are exquisitely sensitive to caspase-mediated cell death, which is regulated by X-linked inhibitor of apoptosis (XIAP), the primary endogenous inhibitor of caspase activation [20]. It is currently unknown when during development these sex differences in ischemic sensitivity arise, and how neonatal hormone exposure contributes to the development of sex-selective cell death mechanisms.

In the present study, we examined the effect of exogenous neonatal androgen exposure on subsequent adult stroke sensitivity. Testicular testosterone production peaks in rat development in the late gestational period (E18) and is high throughout the first few postnatal days. Using a previously established dosing protocol, neonatal male rats were administered exogenous androgens during the first week of life and subjected to middle cerebral artery occlusion (MCAO) in adulthood [21]. Male rats were utilized in this study as we were interested in the effect of excess testosterone on the masculinized and testosterone-sensitive brain during the period of highest brain plasticity. Previous studies have shown that exogenous testosterone in vitro upregulates aromatase mRNA in germ cells [21], which may have a significant impact on the brain's response to ischemic injury. Therefore, this work was undertaken to determine if exogenous testosterone would exacerbate or reduce subsequent ischemic injury in adulthood. Infarct damage, brain and gonadal aromatase levels, and the effect of exogenous neonatal testosterone on cell death programming were evaluated. We specifically avoided early castration in this study to avoid possible preconditioning effects.

\section{Methods}

Animals and Hormone Injections

Male Wistar rats were subcutaneously injected with $0.1 \mathrm{mg}$ testosterone propionate (TP; Sigma, St. Louis, Mo., USA) in $0.05 \mathrm{ml}$ sesame oil or $0.05 \mathrm{ml}$ sesame oil alone for postnatal days 1 through 5 [22], or implanted with a DHT pellet ( $0.5 \mathrm{mg}$, 7-day release) or placebo pellet (Innovative Research of America, Sarasota, Fla., USA) subcutaneously in the scapular area on postnatal day 1. Each treated group was compared to its vehicle-treated cohort. Rats remained in a temperature controlled animal room with $12 \mathrm{~h}$ alternating light and dark cycles until they reached sufficient weight for MCAO (at least $300 \mathrm{~g}$ ) at approximately postnatal day 80 . Three different adult cohorts were utilized in this study: (1) TP and oil 
group for infarct analysis (TP $\mathrm{n}=15$, oil $\mathrm{n}=14$ ); (2) DHT and placebo group for infarct analysis (DHT $n=15$, placebo $n=13$ ), and (3) TP and oil group for protein analysis from brain and gonads (stroke $\mathrm{n}=8$, sham $\mathrm{n}=4$ for both). Serum hormone levels and gonad weights were measured in all animals at sacrifice. An additional neonatal (PND16) TP or oil-treated cohort consisting of 11 animals in each group was used to assess neonatal hormone levels and gonad weights. Two sham and four stroke samples in group 3 were examined for Western blot analysis, and the remaining four stroke and two sham samples were utilized for the brain aromatase activity assay as well as aromatase enzyme-linked immunoassay (ELISA). This study was conducted in accordance with the NIH guidelines for the care and use of animals in research and under protocols approved by the Animal Care and Use Committee of the University of Connecticut Health Center.

\section{Ischemic Model}

Cerebral ischemia was induced by $90 \mathrm{~min}$ of right reversible MCAO model under isoflurane anesthesia as previously described [23]. Rectal temperature was monitored with a MONOTHERM system and maintained at approximately $37^{\circ} \mathrm{C}$ during surgery and ischemia with an automated temperature control feedback system. A midline ventral neck incision was made, and unilateral MCAO was performed by inserting a silicone rubber-coated monofilament (Doccol Corp, Redlands, Calif., USA) into the right internal carotid artery $18 \mathrm{~mm}$ from the internal carotid/pterygopalatine artery bifurcation via an external carotid artery stump. Sham animals underwent the same procedure, but the suture was not advanced into the middle cerebral artery. Laser Doppler flow (Moor Instruments Ltd., Axminster, UK) was measured through the thinned skull over the right temporal fossa. Only the mice whose cerebral blood flow (CBF) showed a drop of over $70 \%$ of baseline just after MCAO were included. Animals were sacrificed at $24 \mathrm{~h}$ after the onset of ischemia for all experimental procedures. Neurologic deficit score was measured at the time of sacrifice $(24 \mathrm{~h})$. The behavior scoring was as follows [24]: 0, no deficit; 1 , forelimb weakness and torso turning to the ipsilateral side when held by tail; 2 , circling to affected side; 3 , unable to bear weight on affected side, and 4 , no spontaneous locomotor activity or barrel rolling.

\section{Histology and Assessment of Infarct}

At $24 \mathrm{~h}$ after stroke/reperfusion, rats were sacrificed with pentobarbital overdose (i.p.). Animals were perfused transcardially with ice-cold PBS followed by $4 \%$ paraformaldehyde; the brain was removed from the skull and post-fixed for $18 \mathrm{~h}$, and subsequently placed in cryoprotectant ( $30 \%$ sucrose). The brain tissue was cut into $30-\mu \mathrm{m}$ free-floating coronal sections on a freezing microtome and every eighth slice was stained by cresyl violet staining for evaluation of ischemic cell damage [25]. The infarct volumes were analyzed using Sigmascan Pro5 and expressed as a percentage of the contralateral hemispheric structure to correct for edema (Swanson's method) as previously described [23]. Analysis was performed by an investigator blinded to treatment group.

\section{Estradiol, Testosterone, DHT and Aromatase ELISAs}

Blood was taken at sacrifice from the right ventricle with heparinized syringes and centrifuged at $6,000 \mathrm{rpm}$ for $10 \mathrm{~min}$ at $4^{\circ} \mathrm{C}$ to yield serum for hormone detection. Serum was stored at $-80^{\circ} \mathrm{C}$ until use. ELISAs were performed using kits for testosterone (Beckman Coulter, Brea, Calif., USA) and 17 $\beta$-estradiol (Bio-
Quant, San Diego, Calif., USA) following the manufacturers' protocol. Male gonads and brains were homogenized according to the manufacturer's protocol for aromatase ELISA (USCNK, Missouri City, Tex., USA).

\section{Brain Aromatase Assay}

Brain tissue was obtained $24 \mathrm{~h}$ after onset of MCAO by rapid removal of brain with subsequent removal of the cerebellum/occipital pole, olfactory/frontal pole, and striatum and hippocampus, leaving the cortex. The hemispheres were separated and flash frozen in 2 -methylbutane and stored at $-80^{\circ} \mathrm{C}$ until use. Aromatase activity was measured using a sensitive in vitro assay that measures the amount of ${ }^{3} \mathrm{H}_{2} \mathrm{O}$ formed during the conversion of a saturating concentration $(0.3 \mu \mathrm{M})$ of [1beta- $3 \mathrm{H}]$ androstenedione to estrone [14].

\section{Protein Analysis}

Brain tissue was obtained at $24 \mathrm{~h}$ after onset of MCAO by rapid removal of brain with subsequent removal of the cerebellum/ occipital pole and olfactory/frontal pole. The samples were flash frozen in 2-methylbutane and stored at $-80^{\circ} \mathrm{C}$ until use. Subcellular fractionation was performed on the ischemic (right) hemisphere as previously described [26] to yield cytosolic fractions. Protein concentrations were determined by bicinchoninic acid assay Protein Assay Kit (Thermo Fisher Scientific Inc., Rockford, Ill., USA) and subjected to Western Blotting as previously described [23]. Briefly, proteins were resolved on SDS-PAGE gradient gels (4-15\%) and transferred to a polyvinylidene difluoride membrane. Blots were probed for XIAP (1:250; BD Biosciences) and $\beta$-tubulin (1:1,000; Abcam) as a cytosolic loading control. Blots were incubated in 5\% nonfat milk in TBST (Tris-buffered saline with $0.1 \%$ Tween 20 ) for $1 \mathrm{~h}$ at room temperature for membrane blocking. Then, the blots were incubated overnight at $4^{\circ} \mathrm{C}$ in primary antibody diluted in 4\% BSA in TBST. Secondary antibodies (goat anti-rabbit IgG 1:5,000 and goat anti-mouse IgG 1:2,000; Santa Cruz) were diluted, and signal was detected with ECL kit (Amersham Biosciences). Densitometry was performed with Photoshop.

\section{Statistics}

Data from individual experiments are presented as the mean \pm SEM and analyzed with a t test when comparing two groups, and two-way ANOVA for the comparison of the means between multiple experimental groups. Neurological deficit scores (NDS) are presented as median (interquartile), and were analyzed with a Mann-Whitney $U$ test. $\mathrm{p}<0.05$ was considered statistically significant. All histological measurements and behavioral assessments were made by investigator blinded to treatment group.

\section{Results}

Physiologic Differences between TP-and DHT-Treated Rats Compared with Vehicle Cohorts

Male rats treated with TP during the neonatal period had similar body weights in adulthood as oil-treated male rats (table 1). However, DHT-implanted male rats were significantly smaller than placebo-implanted males in 
Table 1. Physiological parameters in adult rats

\begin{tabular}{lcclr}
\hline Group & $\begin{array}{l}\text { Rats } \\
\mathrm{n}\end{array}$ & Body weight, g & $\begin{array}{l}\text { Body tempera- } \\
\text { ture, }{ }^{\circ} \mathrm{C}\end{array}$ & $\begin{array}{l}\text { CBF drop } \\
\%\end{array}$ \\
\hline $\mathrm{TP}$ & 15 & $420.9 \pm 0.097$ & $38.3 \pm 0.157$ & $79.2 \pm 1.013$ \\
Oil & 14 & $410.8 \pm 0.054$ & $38.2 \pm 0.141$ & $81.2 \pm 0.717$ \\
DHT & 15 & $391 \pm 0.077^{*}$ & $38.2 \pm 0.079$ & $82 \pm 0.796$ \\
Placebo & 13 & $426.6 \pm 0.053^{*}$ & $38.4 \pm 0.125$ & $81.9 \pm 0.725$ \\
\hline
\end{tabular}

Values are expressed as mean \pm SEM. No significant differences in physiological parameters were seen between groups, except that body weight differed between DHT and placebo groups. ${ }^{*} \mathrm{p}<0.05$.

adulthood (DHT vs. placebo: 391 vs. $426.6 \mathrm{~g} ; \mathrm{n}=15$ and 13 , respectively; $\mathrm{p}<0.001$ ). All animals had similar body temperature during $\mathrm{MCAO}$, which was in the physiological range, and similar reductions in $\mathrm{CBF}$ during both MCAO and early reperfusion as seen by laser Doppler flow in TP versus oil or DHT versus placebo (see table 1).

\section{Differential Effects of Neonatal TP and DHT on \\ Stroke Outcomes}

Neonatal TP-injected and oil vehicle-injected rats were allowed to age until adulthood prior to MCAO. Measurements of infarct volume $24 \mathrm{~h}$ after stroke showed that TP-treated males had significantly smaller infarct volumes than their oil-treated counterparts in both the striatum and overall in the ipsilateral (stroke) hemisphere (fig. 1a, b). Cortical infarct volumes were not significantly different between the groups. In contrast, males treated with DHT had no difference in infarct size as compared to their placebo-treated littermates in any brain area examined (fig. 1a, c). TP rats had lower NDS compared to the oil-treated group $24 \mathrm{~h}$ after stroke, but this difference was not significant [TP vs. oil: $2(1,2)$ vs. $2(1,3)$; data presented as median (1st quartile, 3rd quartile); fig. 1d]. DHT rats had similar NDS compared with vehicle group [DHT vs. vehicle: $2(2,3)$ vs. $2.5(2,3)$; fig. 1e].

\section{Exogenous Neonatal TP but Not DHT Influenced Adult Serum Sex Hormone Levels and Gonadal \\ Weights}

As TP can be converted by aromatase into estradiol, which is neuroprotective in many preclinical stroke models [27], the serum levels of circulating sex hormone levels were assessed. Serum from adult animals was assayed for estradiol and testosterone levels in TP-, DHT-, and vehicle-treated rats. Significantly lower serum testosterone levels were seen in TP-treated rats, which also had a significant elevation in serum estradiol levels compared to oil-treated males (fig. 2a, b). This pattern was also seen in TP-treated sham rats compared to oil-treated shams (sham: TP vs. oil, testosterone $5.9 \mathrm{vs} .14 .2 \mathrm{ng} / \mathrm{ml}$, estradiol 36.2 vs. $14.6 \mathrm{ng} / \mathrm{ml} ; \mathrm{n}=4$ /group). Administration of neonatal DHT did not influence testosterone or estradiol levels at the time of stroke in males (fig. $2 c, d$ ).

Gonadal weights were assessed to determine the end effect of exogenous neonatal testosterone and DHT on peripheral reproductive tissues. As seen previously [28], exposure to androgens in early development had profound effects on the testes. Rats exposed to either neonatal TP or DHT had significantly smaller testes compared to the vehicle-treated group, suggesting similar peripheral effects of both of these androgens on peripheral tissue (fig. 2e, f). Because of the discrepancy in body weights, gonad weights relative to body weights and the gonad/ body weight ratio were assessed. The testes were consistently smaller in the TP and DHT groups when compared to the oil and placebo control groups, respectively (fig. $2 g, h)$.

\section{Neonatal TP Treatment Increased Adult Gonadal Aromatase Levels but Not in the Brain}

To determine the possible mechanisms by which neonatal TP treatment led to an increase in adult serum estradiol levels and subsequent neuroprotection that was not seen after treatment with the non-aromatizable androgen DHT, brain and gonadal aromatase levels were measured. A significant increase in gonadal aromatase was seen in adult TP-treated males compared with oiltreated males (fig. 3a). No differences between groups were seen in brain aromatase levels (fig. 3b) or brain (cortex) aromatase activity (fig. 3c).

\section{Exogenous TP Treatment also Influenced Acute Neonatal Sex Hormone Levels}

Due to the observed robust differences in adult serum hormone levels in the TP-treated rats, we were interested to see if this response was also present in neonates shortly after testosterone injection. PND16 TP-treated male pups had significantly decreased gonad weights compared with oil pups, consistent with what was seen in the adult group (fig. $4 \mathrm{a}$ ), which was consistent when gonad weight was measured relative to body weight (fig. 4b). There were no significant differences in testosterone levels at this time (fig. 4c), but TP-treated pups had significantly decreased estradiol levels compared with the oil group at this early time point (fig. $4 \mathrm{~d}$ ), opposite to what 
Fig. 1. Stroke outcomes in adult rats exposed to neonatal TP or DHT treatment. a Cresyl violet-stained coronal brain slices showing infarct (white) in TP- or DHTtreated rats. $\mathbf{b}$ Quantification of infarct in TP- and oil-injected rats (shown as a percentage of the contralateral structure) in the cortex, striatum, and hemisphere ( $\mathrm{n}=$ 15 for TP males, $\mathrm{n}=14$ for oil males; * $\mathrm{p}<$ 0.05 vs. oil group; ${ }^{* *} \mathrm{p}<0.001$ vs. oil group). c Quantification of infarct in DHT- and placebo-treated rats (shown as a percentage of the contralateral structure) in the cortex, striatum, and hemisphere; $\mathrm{n}=13$ for placebo group, $\mathrm{n}=15$ for DHT group. NDS in TP-treated (d) and DHTtreated (e) groups. Error bars present the minimum or maximum value.
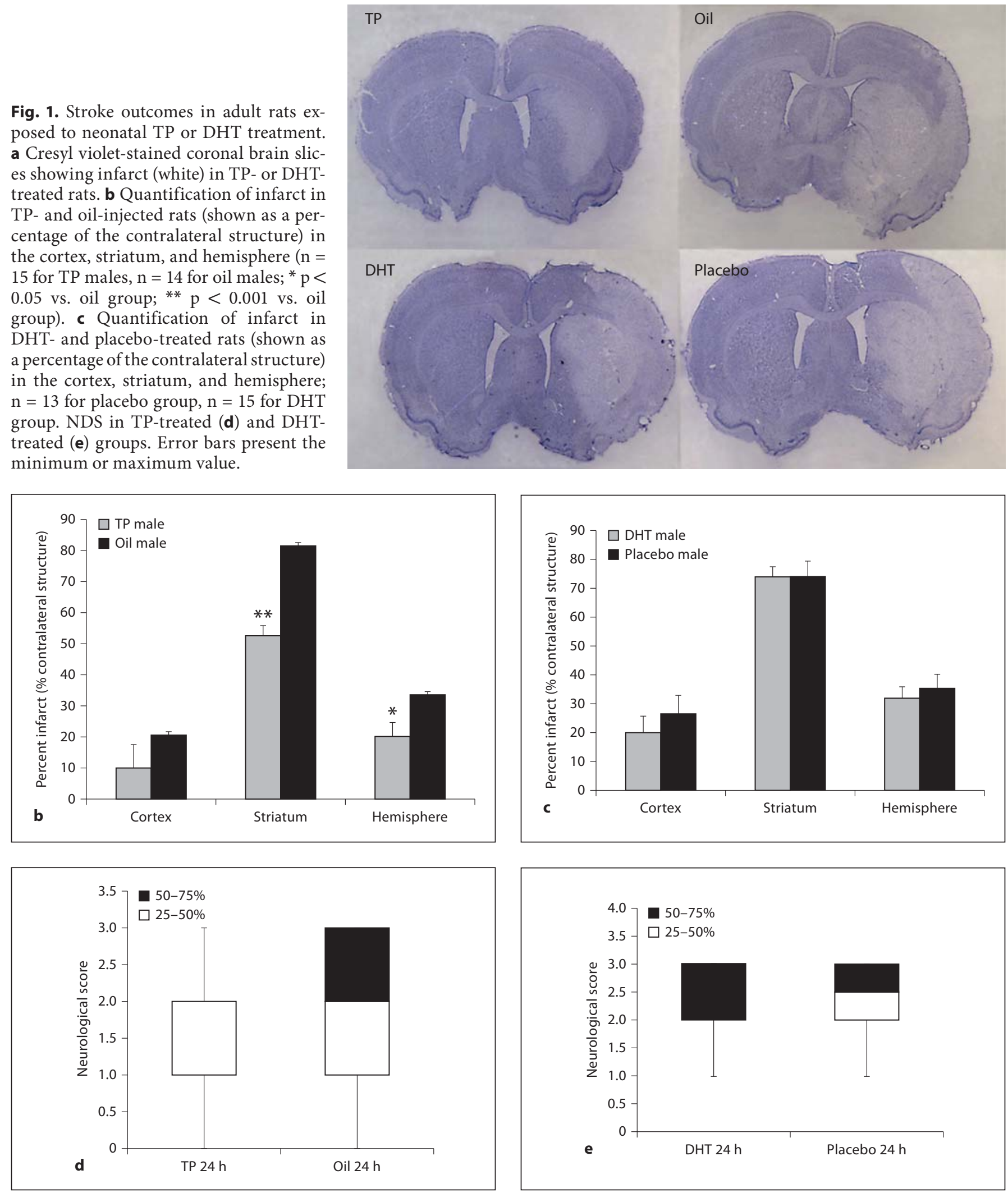

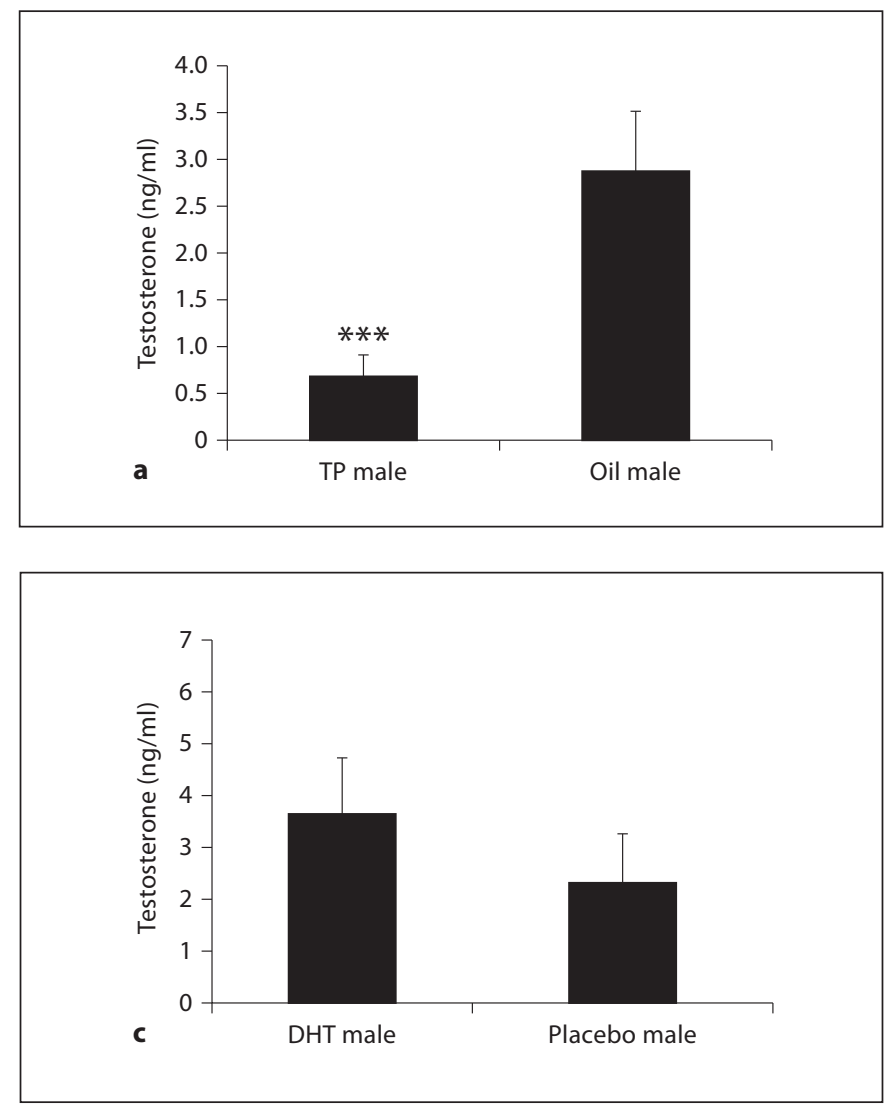

Fig. 2. Serum testosterone and estradiol levels as well as gonadal weights were affected by neonatal testosterone and DHT injection in adult animals. a Serum testosterone levels were increased in oil- compared to TP-treated rats at the time of sacrifice $\left({ }^{* *} \mathrm{p}<\right.$ 0.0001 vs. oil group, $n=10$ /group). b Serum estradiol levels were
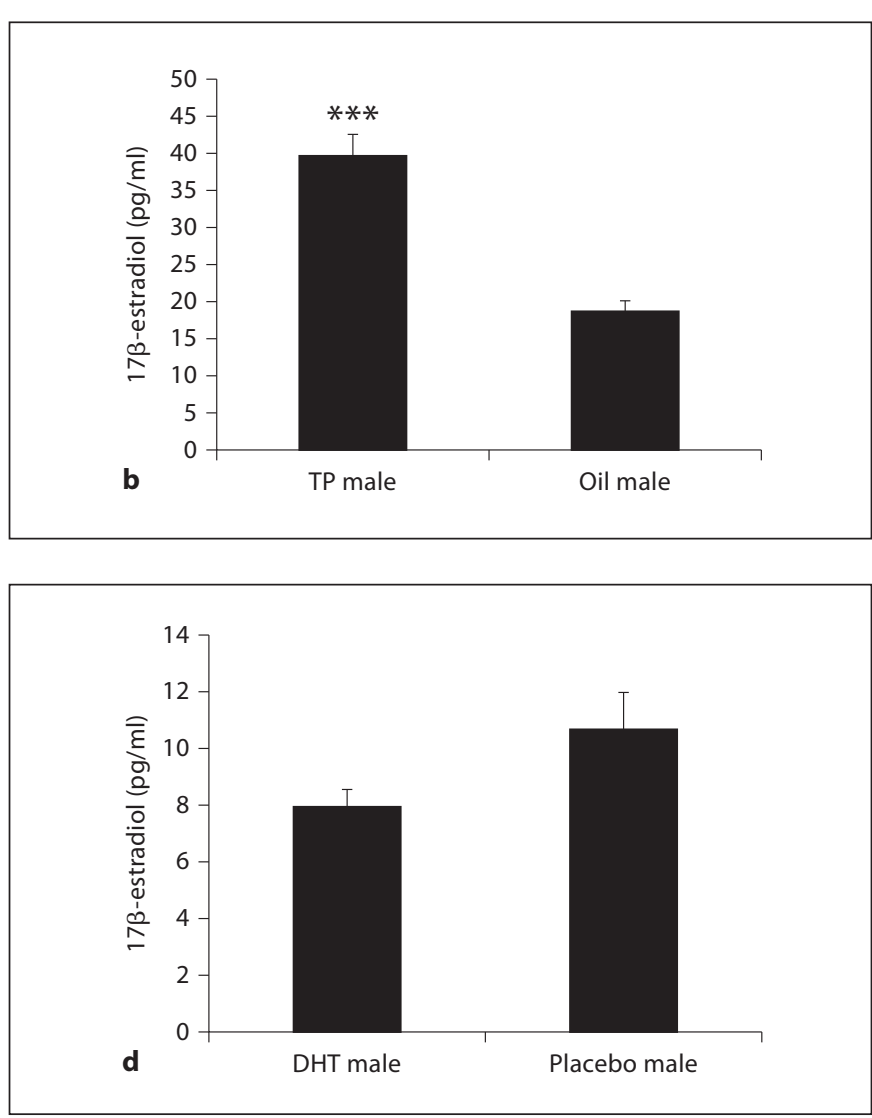

increased in TP- compared with oil-treated male rats at the time of stroke ( ${ }^{* *} \mathrm{p}<0.0001$ vs. TP group, $\mathrm{n}=7$ /group). Serum levels of testosterone (c) and estradiol (d) were not significantly different between DHT- and placebo-pellet-implanted rats at the time of sacrifice $($ DHT $n=15$, placebo $n=13)$. was observed in adults. Interestingly, aromatase levels in the testes of TP-treated pups was also significantly decreased compared with oil-treated pups (fig. 4e), unlike the higher gonadal aromatase levels observed in adulthood. Homogenized brain samples from these rats at PND16 showed no difference in aromatase levels between groups (data not shown).

\section{Sex-Specific Cell Death Pathways May Be Influenced by Exogenous Sex Hormone Treatment}

To determine if neonatal TP administration influenced levels of key sex-specific cell death-signaling molecules, levels of XIAP, the most potent endogenous inhibitor of caspase activation, were directly examined. XIAP levels in adulthood were increased in rats exposed to neonatal TP compared to vehicle-treated controls (fig. $5 \mathrm{a}, \mathrm{b}$ ). Increased XIAP was seen in both sham and stroke TP-treated males, suggesting that the increase in $\mathrm{XIAP}$ is not dependent on injury but was a consequence of treatment with TP.

\section{Discussion}

In this study, we investigated the role of testosterone in the response to stroke. More specifically, we examined how exposure to neonatal androgens can affect the male brain's response to a subsequent ischemic injury in adulthood. Adult females have less damage after experimental stroke, but whether this is secondary to reduced exposure to a detrimental hormone (androgens) or exposure to a potentially beneficial hormone (estradiol) and when this ischemic phenotype develops is unclear. We used a developmental model, as our goal was to explore when sexual 

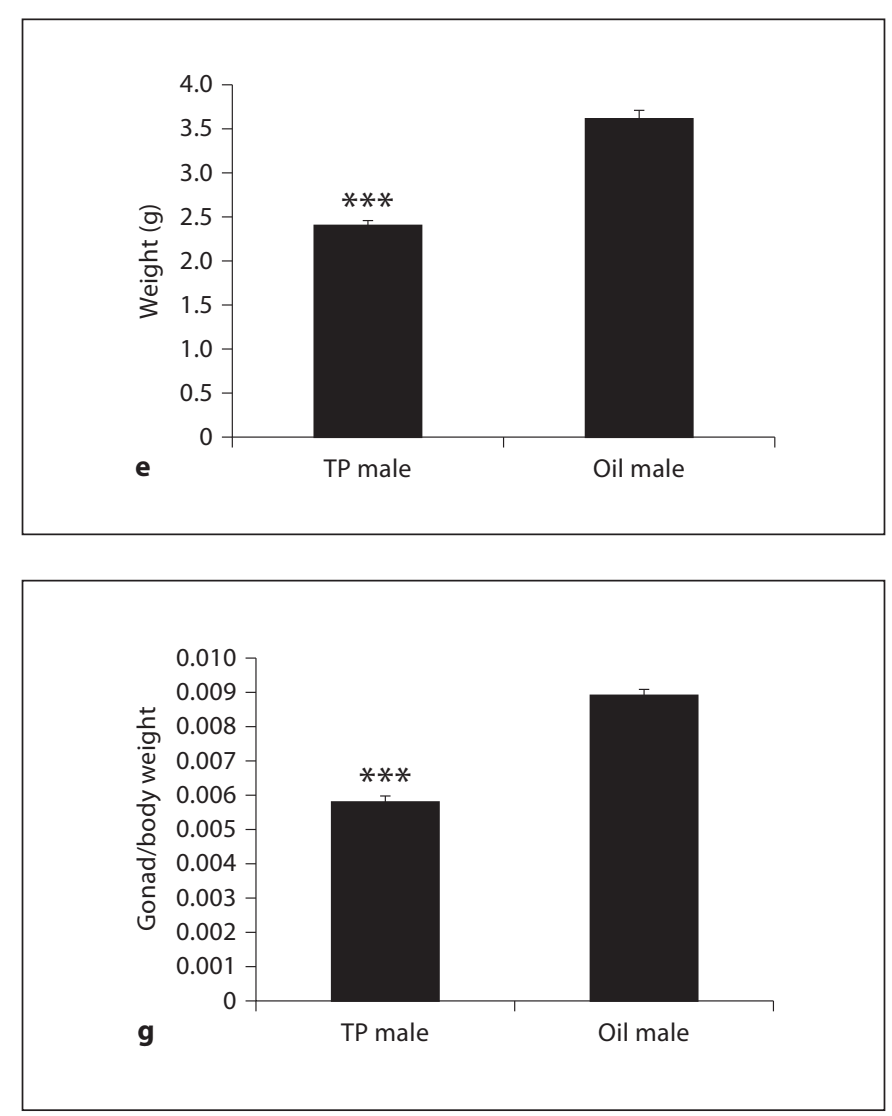

Fig. 2. Serum testosterone and estradiol levels as well as gonadal weights were affected by neonatal testosterone and DHT injection in adult animals. e Gonadal weights were decreased in TP-injected males compared to oil controls $\left({ }^{* *} \mathrm{p}<0.0001\right.$ vs. oil group, $\mathrm{n}=15$ for TP group, $\mathrm{n}=14$ for oil group). $\mathbf{f}$ Adult gonad weights of DHT-implanted male rats were decreased compared to placebo

dimorphism in stroke sensitivity occurs. This study revealed several unexpected and important new findings that elucidate the contribution of early hormonal exposure to adult ischemic sensitivity. Firstly, exposure to exogenous neonatal TP alters the subsequent sensitivity to ischemic injury in adult rats, leading to significant reductions in infarct size. In contrast, neonatal exposure to the non-aromatizable androgen DHT had no effect on infarct volume. The change in infarct volumes with TP administration was not accompanied by improvement of NDS; however, the NDS often does not correlate with infarct size $[20,29,30]$. Secondly, neonatal TP exposure leads to dramatic changes in circulating adult steroid hormone levels, resulting in a decrease in serum testosterone but a significant increase in estradiol levels compared with vehicle-treated controls. Thirdly, a bi-phasic change in testicular aromatase levels was revealed in rats
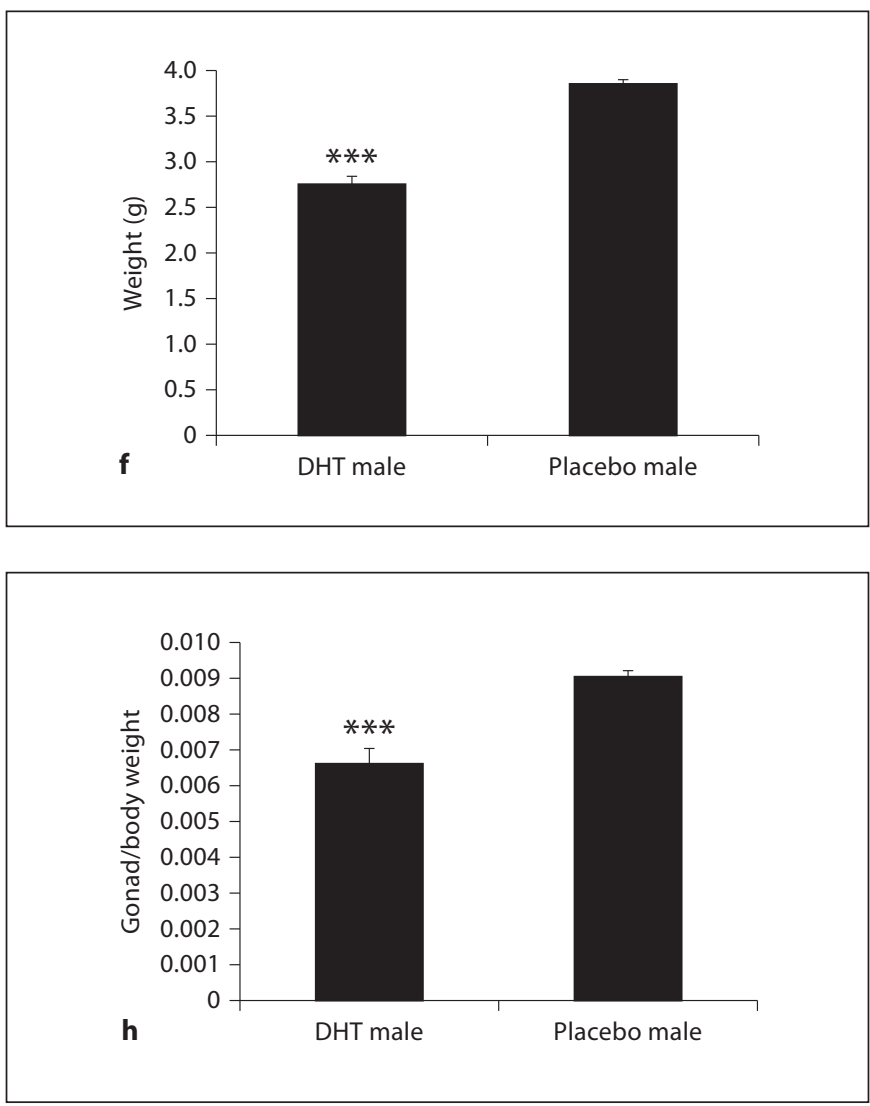

male rats $\left({ }^{* *} \mathrm{p}<0.0001\right.$ vs. placebo group, $\mathrm{n}=15$ for DHT group, $\mathrm{n}=13$ for placebo group). Relative gonad/body weights were decreased in the TP group compared to the oil group $(\mathbf{g})$ as well as in the DHT group compared to the placebo group (h) $\left(^{* * *} \mathrm{p}<\right.$ $0.0001)$.

exposed to neonatal exogenous $\mathrm{TP}$, with a suppression of gonadal aromatase during the acute exposure period (developmental) followed by a significant increase in aromatase levels in adulthood. This increased peripheral aromatase expression likely contributes to the higher serum estradiol levels at the time of stroke. Finally, neonatal TP exposure led to lifelong changes in the levels of XIAP, a key anti-apoptotic protein that regulates cell death in the female brain, providing a possible mechanism for the reduction in infarct damage seen in TP-treated animals. Although the activational effects of gonadal steroids have been well described [31], this is the first report of the consequences of neonatal hormone exposure on adult ischemic stroke sensitivity.

Experimental studies [32, 33] have shown that an increase in testicular androgen production occurs and peaks in the late gestational period, around E18 and con- 


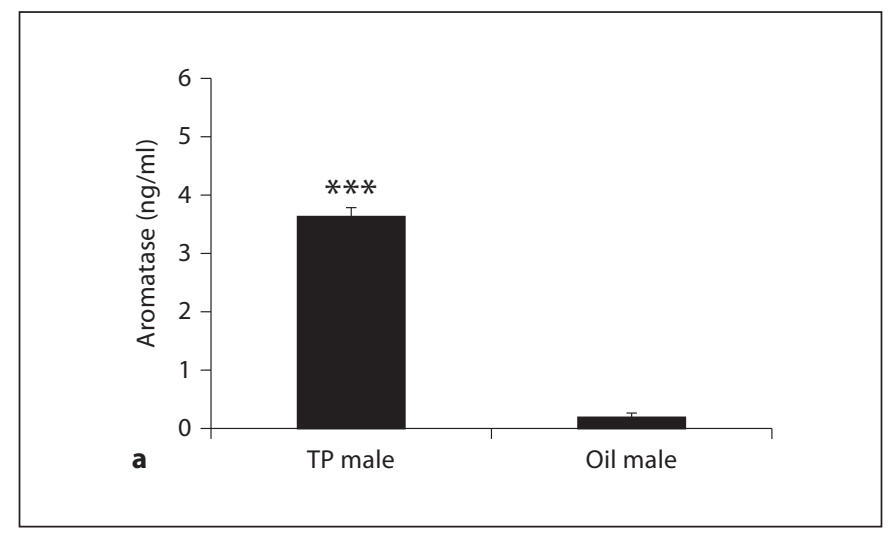

Fig. 3. Increased testicular aromatase levels in TP-injected rats at the time of stroke. a Aromatase levels measured by ELISA in gonads were dramatically increased in TP-injected males compared to oil-injected rats (*** $\mathrm{p}<0.001$ vs. TP group, $n=6$ /group). No differences in aromatase levels (b) and activity (c) in the brain (cortex) between TP- and oil-treated rats at the time of stroke ( $\mathrm{n}=4$ /group).

tinues in the first days of life [34]. Much of this testosterone is aromatized into estradiol and leads to the development of sexually dimorphic areas of the brain. Females do not experience this same increase in circulating estradiol during the perinatal stage due to the lack of testes and high levels of circulating ( $\alpha$-fetoprotein) which binds circulating (serum) estradiol. However, circulating testosterone enters the brain and can be aromatized to produce local estradiol, leading to brain masculinization [35]. To be consistent with what little is known in the literature regarding androgens and brain development, we chose to use a dose that has been previously utilized and reported in the literature [22].

In the present study, neonatal TP and DHT administration led to very different effects on ischemic sensitivity in adult male rats, which is evidently related to changes in adult hormone levels induced by $\mathrm{TP}$ and DHT exposure. The beneficial effects of TP were somewhat unexpected as we initially hypothesized that early TP exposure would worsen ischemic injury based on previous clinical and laboratory data. The neuroprotective effect of early TP exposure led us to examine serum
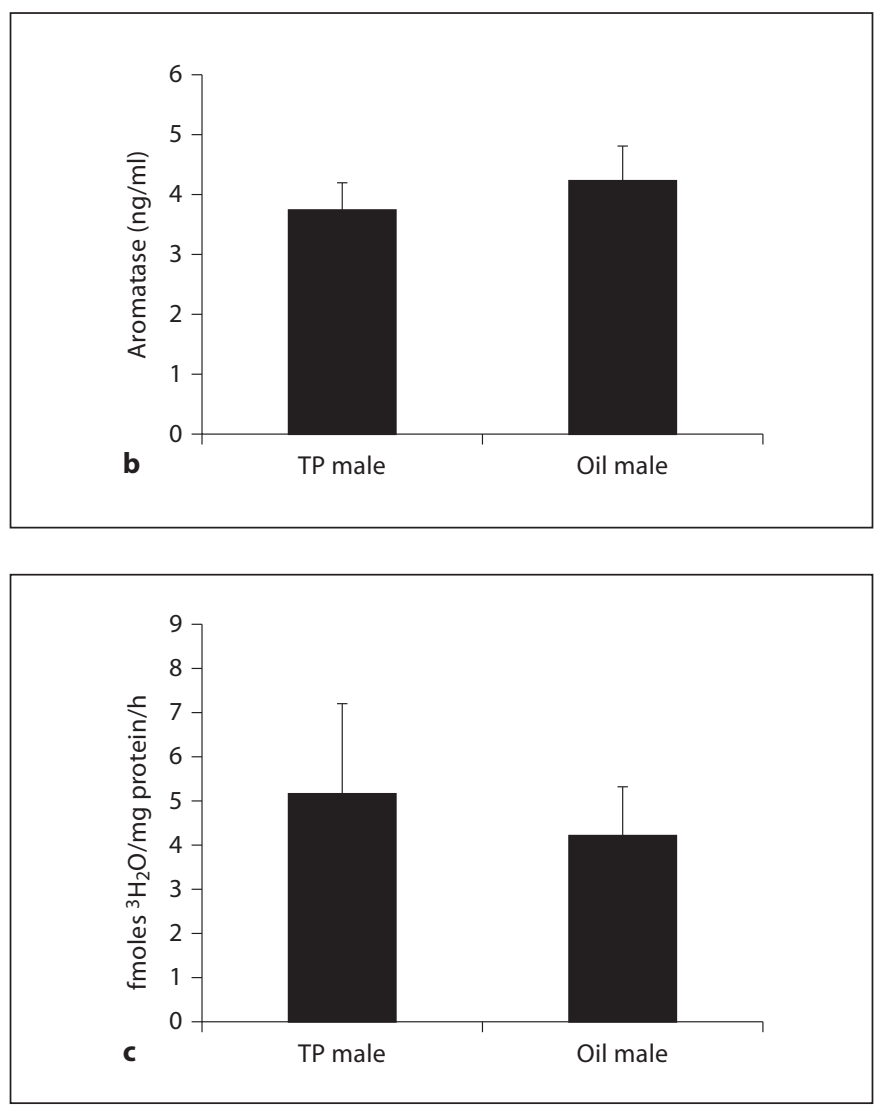

estradiol levels, which were significantly higher in TPtreated rats. As a major source of estradiol is via aromatization of testosterone, and aromatase is known to be involved in ischemic injury, we then examined both serum and brain aromatase levels. Our results demonstrate that excess neonatal testosterone increases aromatase expression peripherally, but not centrally, and results in reduced infarct volumes. Interestingly, this may also explain the observation that TP treatment reduced infarct in the striatum but not in the cortex as the expression of estradiol receptors is lower in the cortex than in the striatum in rodents; therefore, TP treatment would more selectively benefit the striatum if the effect is mediated by aromatization of testosterone into estradiol. However, it should also be noted that the infarct volume in the cortex is much smaller than that in the striatum; therefore, it may be that although estradiol treatment is also neuroprotective in the cortex, but that the cortical infarct was so small in both groups (oil and $\mathrm{TP})$ that treatment did not make a significant difference due to 'floor effects'. 

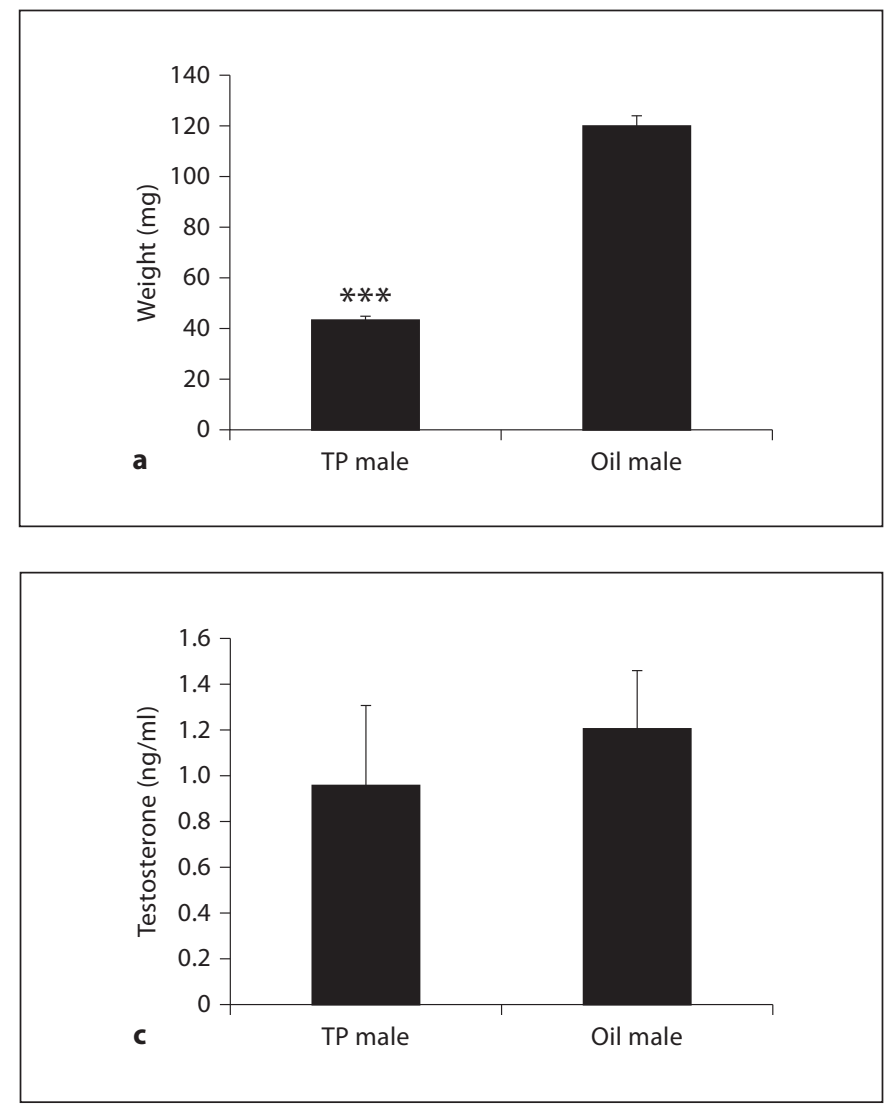

Fig. 4. Neonatal TP administration led to changes in serum hormone levels and gonadal aromatase levels in PND16 rats. TP males had significantly decreased gonad weights (a) as well as gonad/body weights (b) but no difference in testosterone levels (c) compared with vehicle-treated animals. d TP-treated males had decreased serum estradiol levels compared to oil controls at PND16. e Testicular aromatase levels in TP males were significantly decreased compared to oil-treated males $\left({ }^{* * *} \mathrm{p}<0.001\right.$ vs. oil group, $\mathrm{n}=11$ /group).

We cannot exclude a potentially beneficial effect of the significant reduction in serum testosterone in TP-treated males (as seen in fig. 2a), especially as testosterone has been shown to be deleterious in several stroke models [36], and this reduction in serum testosterone was not seen in DHT-treated animals (fig. 2c). Currently, the data on the effects of androgen on ischemic stroke outcomes are conflicting, even in the pre-clinical literature [36] as studies have shown that androgens can either protect or exacerbate ischemic damage depending on the dose used
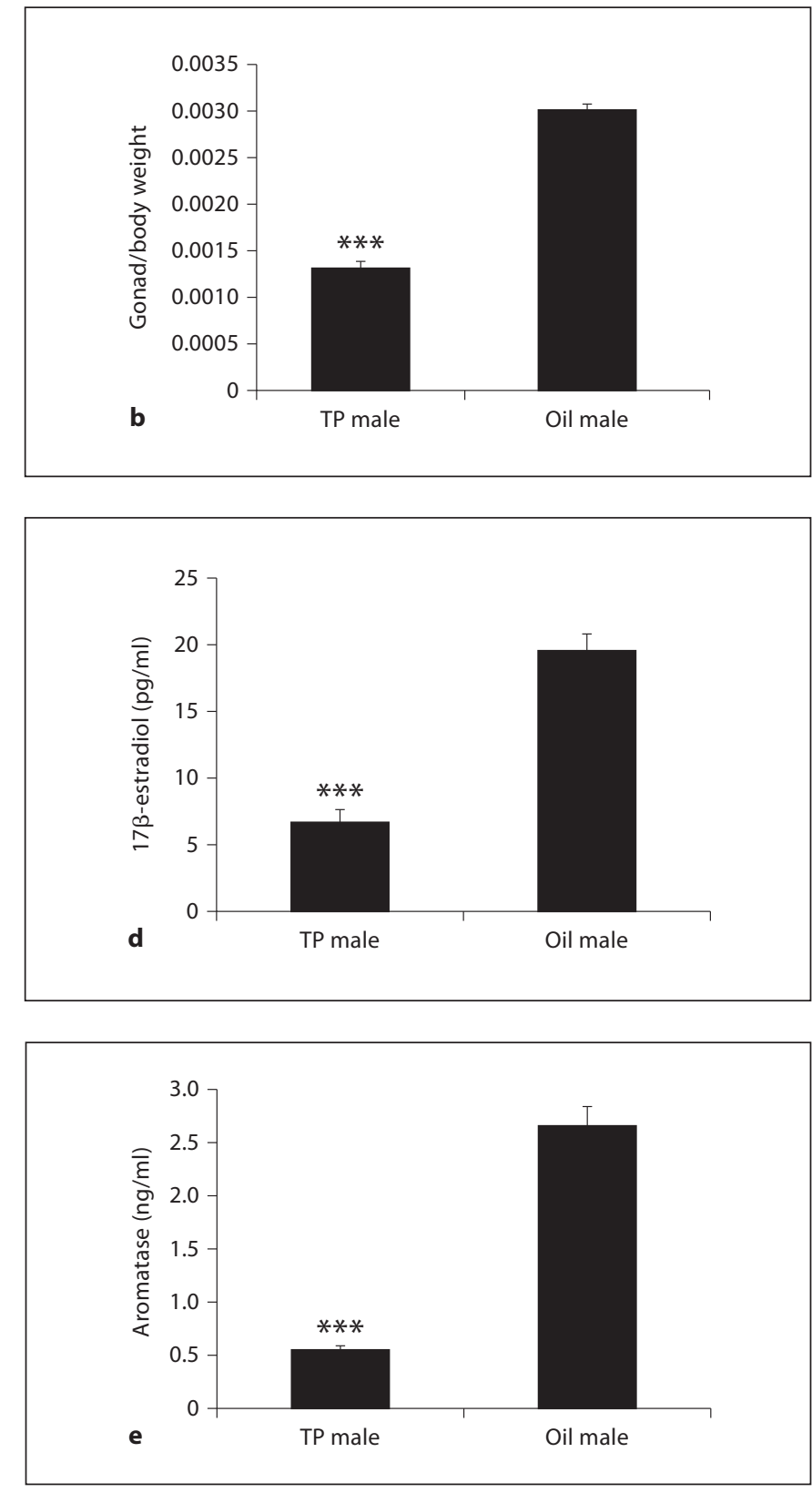

and the age of the animal examined [37]. These uncertainties are reflected in the clinical literature as low circulating testosterone levels are associated with higher stroke incidence and worse outcomes in men over age 70 $[12,13]$ but attempts to replace testosterone led to an increase in vascular risk in elderly men [38]. In pediatric populations, high testosterone appears to be a risk factor for stroke [5], but how much of this risk is from hormonal factors, and how much is from genetic factors (i.e. XY) is not yet known. Similar findings are seen in premature 


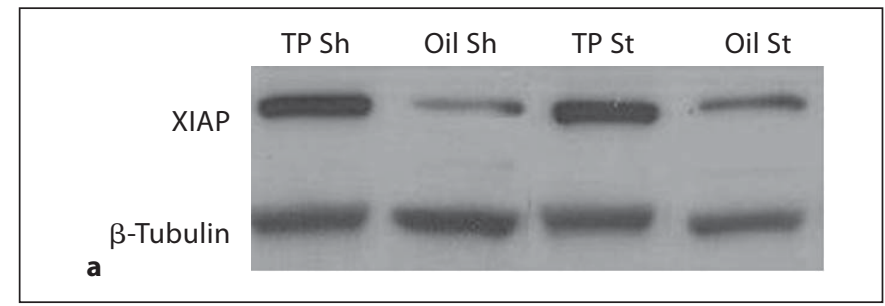

Fig. 5. XIAP levels were increased in adult males exposed to neonatal TP. a Representative Western blots of XIAP protein in sham and stroke rats. $\beta$-Tubulin served as loading control $(\operatorname{sham} n=2$, stroke $\mathrm{n}=4$ ). $\mathbf{b}$ Quantification showed XIAP levels were increased in TP rats both with and without (sham) injury. The optical density was expressed as the ratio of XIAP bands to control bands ( $\beta$-tubulin; ${ }^{* *} p<0.05$ vs. oil groups). Semi-quantitative analysis of densitometry was performed from four independent sets of data.

infants, with males having lower survival and poorer outcomes than females, at a stage of development which is similar to the analogous developmental stage used in these studies [39]. Androgen levels also dramatically drop following both experimental and clinical stroke $[11,40]$ which may contribute to the lower levels seen in epidemiological studies. It is unlikely that the low serum testosterone levels seen in this study were simply secondary to the ischemic injury, as TP-treated sham rats also had significantly decreased testosterone levels compared to oil-treated shams.

Estradiol supplementation decreases infarct volumes in both ovariectomized females and in adult males [27, 41, 42], suggesting that its neuroprotective actions are independent of sex. As neonatal DHT resulted in neither an increase in estradiol nor neuroprotection, we hypothesized that these effects were mediated by TP's actions on aromatase. The significant increase in gonadal aromatase levels in male rats treated with TP suggests a potential mechanism for the increase in serum estradiol. Our results are consistent with a previous in vitro study in germ cells that demonstrated that the addition of testosterone upregulated P450arom mRNA levels in Sertoli Cells and Leydig cells resulting in an increase in estradiol production, an effect not seen after DHT exposure [21]. Genetic deletion of aromatase led to an enhancement in ischemic injury after focal stroke in females lacking aromatase (ARKO), and the detrimental effects of aromatase loss on infarct were significantly more pronounced than seen with the loss of peripheral estradiol with ovariectomy, suggesting an additional effect of local brain estradiol generated by aromatization [43]. Several studies have

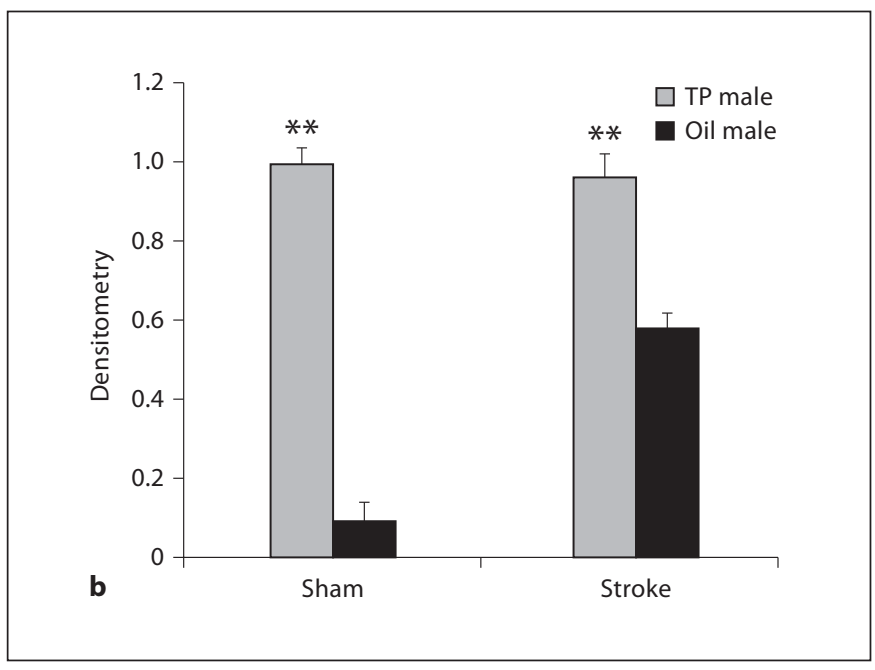

shown that upregulation of brain aromatase occurs after mechanical and ischemic injury [18, 43, 44]. This work suggests that the beneficial effects of neonatal TP exposure on subsequent stroke were mediated by changes in peripheral (gonadal) aromatase expression as no changes were seen in brain aromatase levels (fig. 3b, c). However, these results must be interpreted with caution as there was variability in the samples obtained from brain. We also utilized cortical brain homogenates of the ipsilateral versus the contralateral hemisphere which may have masked small but important changes in aromatase levels in specific areas of the brain. We confirmed the lack of an effect on central aromatase levels with an aromatase assay that utilized the entire hemisphere (minus the occipital pole) which, similar to the cortical activity assay, showed no difference between groups (fig. 3b).

Intriguingly, a different pattern of hormone level changes emerged when serum levels were assessed early in the life of TP-treated rats. In this cohort, there was no significant difference in testosterone levels between the $\mathrm{TP}$ and oil groups, but a robust decrease in estradiol was seen in the TP group compared with oil controls at PND16. Physiologically, there is an increase in estradiol production in the immediate neonatal period which decreases over several days [28]. One previous study directly examined Sertoli cell number and hormone levels in male rats after injection with TP on PND1-5 [28], and found an increase in serum estradiol on PND16 in TPtreated neonates, but lower levels on PND6 compared to controls. Our findings suggest that in the immediate weeks after TP injection, testosterone suppresses peripheral aromatase production, preventing estradiol conver- 
sion; however, as the rat ages, aromatase production in the testes is enhanced and surpasses that of control leading to an enhanced production of estradiol in adulthood. In neonates, TP exposure may decrease Sertoli cell proliferation as well as testosterone production by Leydig cells due to negative feedback on GnRH, FSH and LH which would lead to later testicular atrophy, as was seen at both time points (PND16 and PND80) in this study [45]. Because of these variations in hormones in the neonatal period, it is possible that a hypoxic or ischemic event in the neonatal period, before permanent changes in the hormonal axis occur, would lead to an exacerbation of injury.

In this study, the DHT-treated rats had consistently smaller body weights compared with placebo-treated animals. DHT inhibits the growth of adipocytes, and a growth suppressing effect of DHT has been observed in both male and female chicks at intermediate and high doses $[46,47]$. Interestingly, both DHT and TP rats had equivalently decreased gonad weights compared with their control counterparts regardless of the different body weights; therefore, it is likely that the change in body weight is not contributing to our results and that TP and DHT are exerting the comparable effects on the HPG axis.

Previous work by our lab and others has shown that females are exquisitely sensitive to caspase-induced cell death $[48,49]$, a process mediated by the upstream regulatory protein XIAP [50]. Increased levels of circulating estradiol has been reported to induce the expression of XIAP, leading to enhanced caspase inhibition [51], consistent with our results. Future studies will examine levels of XIAP in DHT-treated animals to confirm this possible estrogenic effect. The increase in XIAP seen in our animals may be responsible for the smaller infarcts as increasing XIAP would lead to a decrease in caspase-induced cell death after injury if the male cell death pathway is 'feminized' by early treatment with TP. This will be investigated in future studies by utilizing a pharmacological inhibitor of XIAP or directly antagonizing estradiol effects with estradiol receptor antagonists.

In conclusion, this study has revealed a novel organizational effect of exogenous androgen exposure on sensitivity to ischemic stroke in the male brain. Neonatal testosterone exposure leads to higher adult serum estradiol levels and subsequent neuroprotection in adulthood, whereas DHT does not, an effect that may be secondary to enhanced aromatization. Neonatal TP exposure also led to changes in sex-specific cell death signaling evidenced by increased expression of XIAP in male rats. This study is a first step towards our understanding of the organizational effects of gonadal steroids and the developmental basis of adult cerebrovascular risk. Understanding these pathways may lead to the development of more efficacious neuroprotective agents and enhance prevention efforts to those at high risk, hopefully leading to reduction in the burden of stroke in all ages.

\section{Acknowledgements}

This work was supported by NI/NINDS (R21NS066406 and R01NS055215 to L.D.M.), The University of Connecticut Health Center Student Research fund (to R.W.P.), and the American Heart association (SDG to F.L.).

\section{References}

-1 Roger VL, Go AS, Lloyd-Jones DM, et al: Heart disease and stroke statistics - 2011 update: a report from the American Heart Association. Circulation 2011;123:e18-e209.

$\checkmark 2$ Appelros P, Stegmayr B, Terent A: Sex differences in stroke epidemiology: a systematic review. Stroke 2009;40:1082-1090.

$\checkmark 3$ Persky RW, Turtzo LC, McCullough LD: Stroke in women: disparities and outcomes. Curr Cardiol Rep 2010;12:6-13.

4 Normann S, de Veber G, Fobker M, et al: Role of endogenous testosterone concentration in pediatric stroke. Ann Neurol 2009;66:754758

5 Vannucci SJ, Hurn PD: Gender differences in pediatric stroke: is elevated testosterone a risk factor for boys? Ann Neurol 2009;66: 713-714.
-6 Liu F, Yuan R, Benashski SE, McCullough LD: Changes in experimental stroke outcome across the life span. J Cereb Blood Flow Metab 2009;29:792-802.

$>7$ Du L, Bayir H, Lai Y, et al: Innate genderbased proclivity in response to cytotoxicity and programmed cell death pathway. J Biol Chem 2004;279:38563-38570.

8 Kawamata T, Alexis NE, Dietrich WD, Finklestein SP: Intracisternal basic fibroblast growth factor (bFGF) enhances behavioral recovery following focal cerebral infarction in the rat. J Cereb Blood Flow Metab 1996;16:542-547.

$\checkmark 9$ Liu M, Dziennis S, Hurn PD, Alkayed NJ: Mechanisms of gender-linked ischemic brain injury. Restor Neurol Neurosci 2009; 27:163-179.
Cheng J, Alkayed NJ, Hurn PD: Deleterious effects of dihydrotestosterone on cerebral ischemic injury. J Cereb Blood Flow Metab 2007;27:1553-1562.

11 Uchida M, Palmateer JM, Herson PS, DeVries AC, Cheng J, Hurn PD: Dose-dependent effects of androgens on outcome after focal cerebral ischemia in adult male mice. J Cereb Blood Flow Metab 2009;29:14541462.

12 Yeap BB, Hyde Z, Almeida OP, et al: Lower testosterone levels predict incident stroke and transient ischemic attack in older men. J Clin Endocrinol Metab 2009;94:2353-2359. 
$\checkmark 13$ Hollander M, Koudstaal PJ, Bots ML, Grobbee DE, Hofman A, Breteler MM: Incidence, risk, and case fatality of first ever stroke in the elderly population. The Rotterdam Study. J Neurol Neurosurg Psychiatry 2003; 74:317-321.

14 Roselli CE, Ellinwood WE, Resko JA: Regulation of brain aromatase activity in rats. Endocrinology 1984;114:192-200.

15 Roselli CE, Liu M, Hurn PD: Brain aromatization: classic roles and new perspectives. Semin Reprod Med 2009;27:207-217.

16 Lephart ED: A review of brain aromatase cytochrome P450. Brain Res Brain Res Rev 1996;22:1-26.

17 Bardet SM, Cornil CA, Balthazart J: Testosterone recruits new aromatase-imunoreactive cells in neonatal quail brain. Neuroreport 2010;21:376-380.

$\rightarrow 18$ Carswell HV, Dominiczak AF, Garcia-Segura LM, Harada N, Hutchison JB, Macrae IM: Brain aromatase expression after experimental stroke: topography and time course. J Steroid Biochem Mol Biol 2005;96:89-91.

19 Lang JT, McCullough LD: Pathways to ischemic neuronal cell death: are sex differences relevant? J Transl Med 2008;6:33.

20 Grabowski M, Brundin P, Johansson BB: Paw-reaching, sensorimotor, and rotational behavior after brain infarction in rats. Stroke 1993;24:889-895.

21 Bourguiba S, Genissel C, Lambard S, Bouraima H, Carreau S: Regulation of aromatase gene expression in Leydig cells and germ cells. J Steroid Biochem Mol Biol 2003;86:335-343.

-22 Rosen GD, Herman AE, Galaburda AM: Sex differences in the effects of early neocortical injury on neuronal size distribution of the medial geniculate nucleus in the rat are mediated by perinatal gonadal steroids. Cereb Cortex 1999;9:27-34.

-23 McCullough LD, Zeng Z, Blizzard KK, Debchoudhury I, Hurn PD: Ischemic nitric oxide and poly (ADP-ribose) polymerase-1 in cerebral ischemia: male toxicity, female protection. J Cereb Blood Flow Metab 2005;25: 502-512.

-24 Thulin T, Fagher B, Grabowski M, Ryding E, Elmqvist D, Johansson BB: Cerebral blood flow in patients with severe hypertension, and acute and chronic effects of felodipine. J Hypertens 1993;11:83-88.

-25 Li X, Blizzard KK, Zeng Z, DeVries AC, Hurn PD, McCullough LD: Chronic behavioral testing after focal ischemia in the mouse: functional recovery and the effects of gender. Exp Neurol 2004;187:94-104.

-26 Liu F, Li Z, Li J, Siegel C, Yuan R, McCullough LD: Sex differences in caspase activation after stroke. Stroke 2009;40:1842-1848.
27 McCullough LD, Hurn PD: Estrogen and ischemic neuroprotection: an integrated view. Trends Endocrinol Metab 2003; 14: 228-235.

28 Walczak-Jedrzejowska R, SlowikowskaHilczer J, Marchlewska K, Oszukowska E, Kula K: Administration of testosterone inhibits initiation of seminal tubule growth and decreases Sertoli cell number in the earliest period of rat's postnatal development. Folia Histochem Cytobiol 2009;47:S149S154.

29 Alexis NE, Back T, Zhao W, Dietrich WD, Watson BD, Ginsberg MD: Neurobehavioral consequences of induced spreading depression following photothrombotic middle cerebral artery occlusion. Brain Res 1996;706: 273-282.

-30 Liu F, Benashski SE, Persky R, Xu Y, Li J, McCullough LD: Age-related changes in AMPactivated protein kinase after stroke. Age (Dordr) 2012;34:157-168.

- 31 de Vries GJ, Sodersten P: Sex differences in the brain: the relation between structure and function. Horm Behav 2009;55:589-596.

32 Carter MQ, Xue K, Brandl MT, et al: Functional metagenomics of Escherichia coli O157:H7 interactions with spinach indigenous microorganisms during biofilm formation. PLoS One 2012;7:e44186.

- 33 Nardo L, Alizai H, Virayavanich W, et al: Lumbosacral transitional vertebrae: association with low back pain. Radiology 2012, Epub ahead of print.

34 Ishii H, Sato S, Yin C, Sakuma Y, Kato M: Cetrorelix, a gonadotropin-releasing hormone antagonist, induces the expression of melatonin receptor 1a in the gonadotropinreleasing hormone neuronal cell line GT1-7. Neuroendocrinology 2009;90:251-259.

-35 Liu FL, Sheng QS, Chen WB, Wang JH, Hua HJ, Lin JJ: Hand-assisted laparoscopic surgery in radical resection of rectal cancer (in Chinese). Zhonghua Wai Ke Za Zhi 2012;50: 622-624.

36 Jiang ZW, Zhao K, Wang G, et al: Application of surgical robotic system in patients with gastric cancer: a report of 120 cases (in Chinese). Zhonghua Wei Chang Wai Ke Za Zhi 2012; 15:801-803.

37 Cheng J, Hurn PD: Sex shapes experimental ischemic brain injury. Steroids 2010;75:754759.

38 Basaria S, Coviello AD, Travison TG, et al: Adverse events associated with testosterone administration. N Engl J Med 2010;363:109122.

39 Yuan F, Song L, Liu F, Gu L, Zhang Y: Eukaryotic DNA mismatch repair in vitro. Methods Mol Biol 2012;920:149-162.
40 Dash RJ, Sethi BK, Nalini K, Singh S: Circulating testosterone in pure motor stroke. Funct Neurol 1991;6:29-34.

41 Manwani B, McCullough LD: Sexual dimorphism in ischemic stroke: lessons from the laboratory. Womens Health (Lond Engl) 2011;7:319-339.

42 Liu F, Benashski SE, Xu Y, Siegel M, McCullough LD: Effects of chronic and acute oestrogen replacement therapy in aged animals after experimental stroke. J Neuroendocrinol 2012;24:319-330.

43 McCullough LD, Blizzard K, Simpson ER, $\mathrm{Oz}$ OK, Hurn PD: Aromatase cytochrome P450 and extragonadal estrogen play a role in ischemic neuroprotection. J Neurosci 2003; 23:8701-8705.

44 Lavaque E, Mayen A, Azcoitia I, Tena-Sempere M, Garcia-Segura LM: Sex differences, developmental changes, response to injury and cAMP regulation of the mRNA levels of steroidogenic acute regulatory protein, cytochrome p450scc, and aromatase in the olivocerebellar system. J Neurobiol 2006;66:308318.

45 Konkle AT, McCarthy MM: Developmental time course of estradiol, testosterone, and dihydrotestosterone levels in discrete regions of male and female rat brain. Endocrinology 2011;152:223-235.

46 Duskova M, Pospisilova H: The role of nonaromatizable testosterone metabolite in metabolic pathways. Physiol Res 2011;60: 253-261.

47 Fennell MJ, Scanes CG: Inhibition of growth in chickens by testosterone, 5 alpha-dihydrotestosterone, and 19-nortestosterone. Poult Sci 1992;71:357-366.

48 Renolleau S, Fau S, Goyenvalle C, et al: Specific caspase inhibitor Q-VD-OPh prevents neonatal stroke in P7 rat: a role for gender. J Neurochem 2007;100:1062-1071.

49 Renolleau S, Fau S, Goyenvalle C, CharriautMarlangue C: Sex, neuroprotection, and neonatal ischemia. Dev Med Child Neurol 2007;49:477, author reply 477-478.

-50 Siegel C, Li J, Liu F, Benashski SE, McCullough LD: miR-23a regulation of $\mathrm{X}$ linked inhibitor of apoptosis (XIAP) contributes to sex differences in the response to cerebral ischemia. Proc Natl Acad Sci USA 2011;108:11662-11667.

51 Mendonca DF, Chammas R, Liu FT, et al: The inactive form of glycogen synthase kinase-3beta is associated with the development of carcinomas in galectin-3 wild-type mice, but not in galectin-3-deficient mice. Int J Clin Exp Pathol 2012;5:547-554. 